\title{
Right coronary artery fistula misdiagnosed as right atrial cardiac myxoma: A case report
}

\author{
BING WEN ${ }^{1}$, JUNYA YANG ${ }^{2}$, ZHOUYANG JIAO $^{1}$, GUOWEI FU ${ }^{1}$ and WENZENG ZHAO ${ }^{1}$ \\ ${ }^{1}$ Department of Cardiovascular Surgery, The First Affiliated Hospital of Zhengzhou University; \\ ${ }^{2}$ Department of Dermatology, The Fifth Affiliated Hospital of Zhengzhou University, Zhengzhou, Henan 450052, P.R. China
}

Received December 29, 2014; Accepted January 19, 2016

DOI: $10.3892 / \mathrm{ol} .2016 .4457$

\begin{abstract}
The current study describes a case of right coronary artery fistula (CAF) misdiagnosed as right atrial myxoma (RAM). A 33-year-old man presented with a 13-year history of intermittent chest pain, and aggravation for 3 days. Echocardiography revealed an occupying lesion in the right atrium producing a partial dynamic tricuspid obstruction. The initial diagnosis was RAM, which causes partial right ventricular inflow tract obstruction. During cardiopulmonary bypass surgery, a giant mass was detected in the anterior wall of the right ventricle and an abnormal vascular fistula was observed at the bottom of the mass. Successful excision of the mass and closure of the fistula completely relieved the patient's presenting symptoms. The disease was subsequently diagnosed as right CAF draining to the myocardial void. The surgical management and misdiagnosis of the case are discussed herein.
\end{abstract}

\section{Introduction}

Coronary artery fistulas (CAFs) are rare coronary anomalies in which abnormal connections exist between a coronary artery and a cardiac chamber or a major vessel $(1,2)$. CAFs are present in $0.002 \%$ of the global population and comprise $48.7 \%$ of all congenital coronary anomalies. The fistula may be large ( $>250 \mathrm{~mm}$ ) and tends to enlarge over time (3). According to the American College of Cardiology/American Heart Association guidelines (4), percutaneous or surgical closure is strongly recommended for the treatment of a large fistula. Atrial myxomas are the most prevalent type of primary heart tumor, and present with nonspecific symptoms of right heart failure, syncope, exertional dyspnea and pulmonary embolism $(5,6)$. Cardiac myxomas most frequently arise in the left atrium (85\%), and rarely in the right atrium (10\%) or the ventricles (5\%). Although this tumor is histologically benign,

Correspondence to: Dr Bing Wen, Department of Cardiovascular Surgery, The First Affiliated Hospital of Zhengzhou University, 1 Jianshe East Road, Zhengzhou, Henan 450052, P.R. China

E-mail: bingwencn@163.com

Key words: right atrial myxoma, right coronary artery fistula, misdiagnosis it should be classified as a potentially fatal tumor due to the potential for embolic complications, and the standard treatment for cardiac myxoma is surgical removal (7). The present report describes the case of a patient with right CAF draining to the myocardial void, which was misdiagnosed as right atrial myxoma (RAM).

\section{Case report}

A 33-year-old man was admitted to The First Affiliated Hospital of Zhengzhou University for intermittent chest pain. The patient had a 13-year history of chest pain accompanied by a sense of impending doom following activity, which was relieved by rest. The duration, severity and frequency of the pain was aggravated 3 days prior to hospitalization.

Upon physical examination, the patient's heart rate was regular at $50 \mathrm{bpm}$, blood pressure was $112 / 70 \mathrm{mmHg}$ and body temperature was $36.8^{\circ} \mathrm{C}$. The lips and fingernails were neither cyanotic nor clubbed. No thrills were detected in the precordial region. Heart and lung sounds were normal, with no dry or moist rales and no arrhythmia or cardiac murmurs.

All routine laboratory data were within normal limits. A chest X-ray (Multix Fusion; Siemens AG, Munich, Germany) indicated cardiomegaly, predominantly on the right side. In addition, electrocardiography (ECG; 6951E; Nihon Kohden, Tokyo, Japan) demonstrated right atrial enlargement, ST-segment depression on the right precordial ECG and sinus bradycardia, with a heart rate of $51 \mathrm{bpm}$. Echocardiography (Vivid 3; GE Vingmed Ultrasound, Horten, Norway) revealed an occupying mass in the right atrium producing a partial dynamic tricuspid obstruction (Fig. 1). Further complete catheterization was not conducted due to the risk of causing pulmonary embolization. The condition was diagnosed as RAM, which typically causes partial right ventricular inflow tract obstruction, based on the presence of the occupying mass in the right atrium on echocardiography (8).

Following preoperative preparation, the patient was taken for cardiopulmonary bypass surgery (CPB). The patient's hemodynamics, blood oxygen saturation, ECG, body temperature and urine volume were monitored. The heart was approached through median sternotomy and CPB was performed. During surgery, the right atrium was opened; however, no occupying mass was found in the right atrium. Unexpectedly, a giant cyst was located in the anterior wall of right ventricle, the 


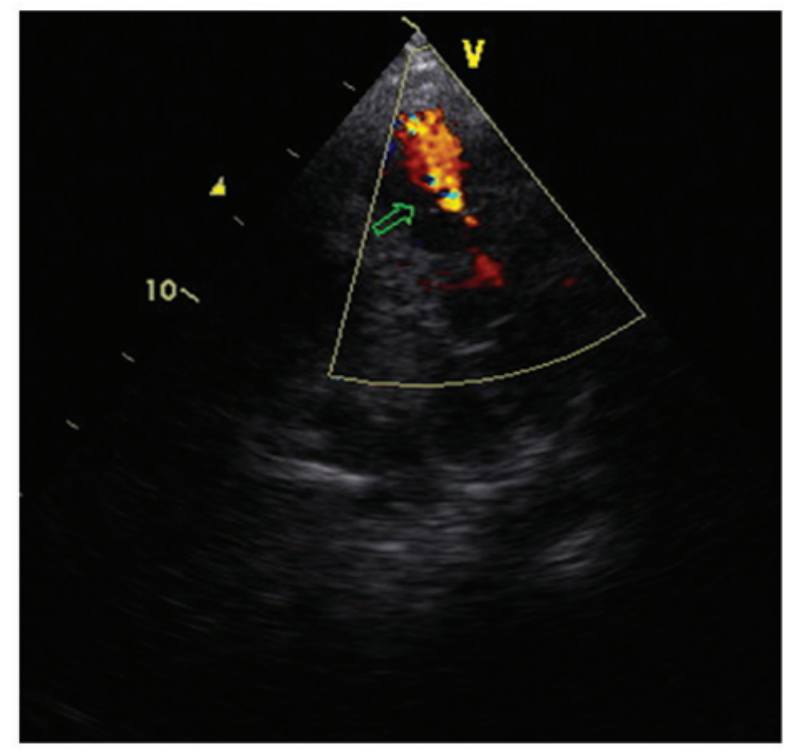

Figure 1. Transthoracic echocardiography showing an occupying mass in the right atrium producing a partial dynamic tricuspid obstruction.

posterior part of which was attached to the anterior tricuspid annulus (Fig. 2). In order to acquire a pathological diagnosis of the mass, a quick frozen pathological section was rapidly analyzed, and the pathological result indicated the presence of myocardium, fibrous tissue and thrombus.

The hemorrhagic cyst was removed intact, and was found to be filled with old thrombus and dark-gray necrotic tissues (Fig. 3). An abnormal vascular fistula of $\sim 8 \mathrm{~mm}$ in diameter was discovered at the bottom of the cyst. To clarify the fistulous communication, blood cardioplegic solution was injected into the right coronary sinus and was observed to overflow from the vascular fistula. Therefore, the patient was diagnosed with right CAF draining to the myocardial void, and neither the right atria or the right ventricle. The fistula was sutured directly with a double-ended needle using an interrupted mattress suture technique. No palpable thrill was detected subsequently. No changes in baseline ECG, blood pressure, pulse rate or blood oxygen saturation were observed during this period. The patient was brought to the cardiac intensive care unit and treated with diuresis and nitroglycerin for blood vessel expansion. Ten days after the operation, the patient was discharged from the hospital. Re-examination one month later revealed no residual fistula flow.

The post-operative pathological findings revealed a hemorrhagic cyst measuring 10x8x8 $\mathrm{mm}^{3}$ and weighing $98 \mathrm{~g}$ (Fig. 3). Resected tissues were frozen, cut into $5-\mu \mathrm{m}$ sections and fixed in acetone (Wuhan Boster Biological Technology, Ltd., Wuhan, China) for histological analysis. Hematoxylin-eosin (Wuhan Boster Biological Technology, Ltd.) staining identified that the outer layer of the sample was tissue, not a blood clot; myocardium, fibrous tissue and thrombus were also observed. For further detection, horseradish-peroxidase (HRP) immunohistochemical staining was performed. Fresh tissue was frozen in liquid nitrogen, embedded in optimal cutting temperature compound (Tissue-Tek O.C.T compound; Sakura Finetek U.S.A., Inc., Torrance, CA, USA) and then cut into cryostat sections (4-8 $\mu \mathrm{m}$ thick). Prior to staining, the slide was

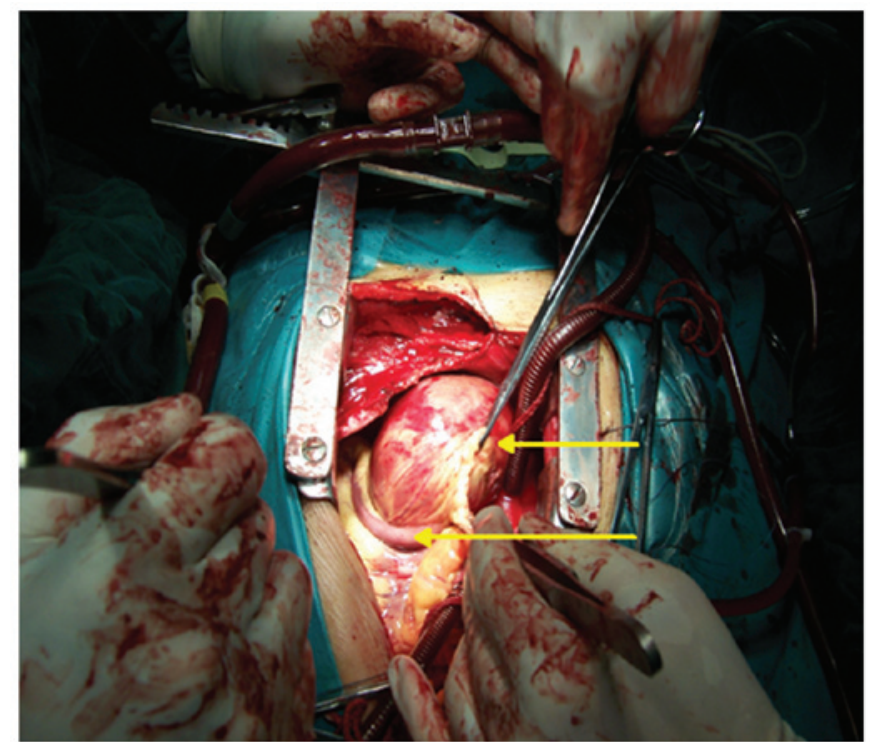

Figure 2. Brief demonstration of the surgical anatomy, exposing a giant cyst in the anterior wall of right ventricle (upper arrow) and the giant right coronary artery (lower arrow).

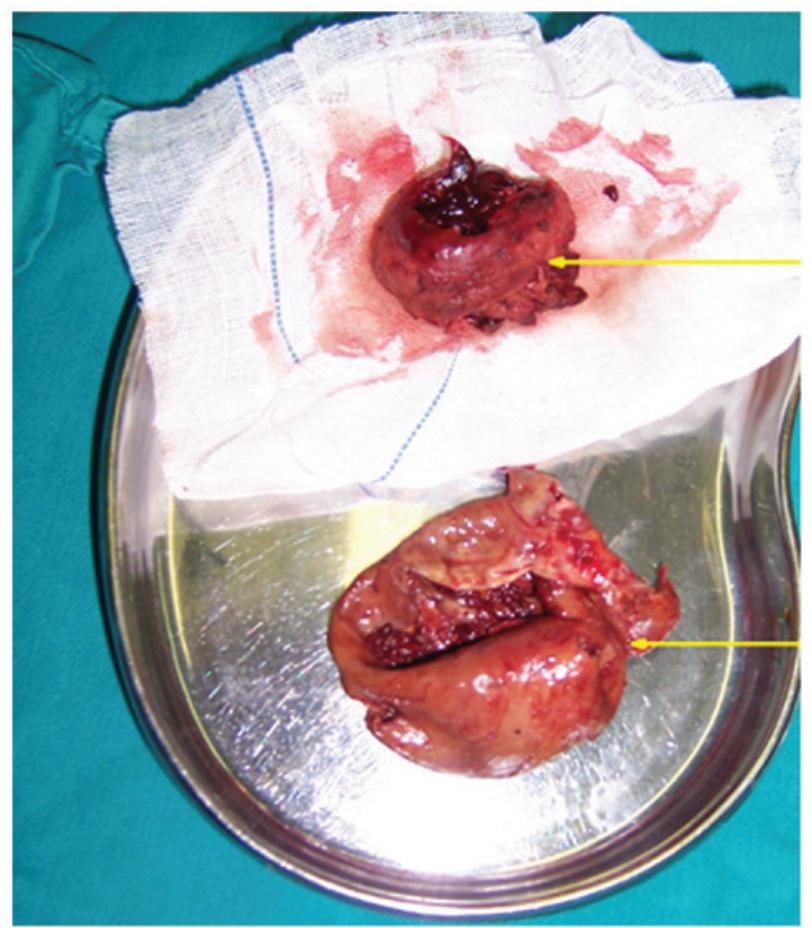

Figure 3. Macroscopic view showing a hemorrhagic cyst measuring $10 \times 8 \times 8 \mathrm{~mm}^{3}$, the thrombus (upper arrow) and the cyst (lower arrow).

warmed to room temperature, fixed in ice-cold acetone and air dried. After rinsing, the sections were incubated with normal serum (Wuhan Boster Biological Technology, Ltd.) followed by primary goat polyclonal fibroblast-specific protein-1 (FSP-1) antibody (diluted 1:100 with antibody dilution buffer; cat no. sc-49158; Santa Cruz Biotechnology Inc., Dallas, TX, USA) for $1 \mathrm{~h}$ at room temperature. After rinsing with phosphate-buffered saline (PBS; Wuhan Boster Biological Technology, Ltd.), the sections were incubated with peroxidase blocking solution [Tiangen Biotech (Beijing) Co., Ltd., Beijing, China) followed 
by mouse anti-rabbit monoclonal $\operatorname{IgG}$ secondary antibody (cat no. M0809-1; 1:500; Hangzhou Hua'an Medical \& Health Instruments Co., Ltd., Hangzhou, China) for half $1 \mathrm{~h}$. After rinsing three times with PBS, the sections were incubated with streptavidin-HRP [Tiangen Biotech (Beijing) Co., Ltd.] in PBS for $30 \mathrm{~min}$, rinsed again, incubated in 3,3'-diaminobenzidine solution (Wuhan Boster Biological Technology, Ltd.), and then rinsed in PBS and distilled water. The sections were subsequently dehydrated through $95 \%$ ethanol for $2 \mathrm{~min}, 100 \%$ ethanol for $3 \mathrm{~min}$, and then cleared in xylene (Wuhan Boster Biological Technology, Ltd.) for $5 \mathrm{~min}$. Finally, coverslips were mounted with mounting medium (Wuhan Boster Biological Technology, Ltd.). Staining was visualized using a microscope (ECLIPSE E400; Nikon Corporation, Tokyo, Japan).

\section{Discussion}

CAF is a rare heart disease. The incidence rate of CAF is estimated to be 1 in 50,000 live births, and it is detected in $\sim 0.2 \%$ of the adult population (9). The majority of these fistulas are congenital, originating from the right coronary artery, and $>50 \%$ drain into a right-sided cardiac chamber (10). CAF may present with heart murmur, congestive heart failure or angina $(11,12)$. Endocarditis may also occur in an untreated coronary fistula (13). Although there are many reports of congenital right $\mathrm{CAF}$, a fistula draining to the myocardial void and forming a hemorrhagic cyst appears to be an extremely rare phenomenon $(14,15)$.

Systolic and diastolic murmurs are common clinical manifestations of CAF $(16,17)$. However, in the present case, no cardiac murmurs were noted on physical examination. ECG as a chief adjuvant diagnostic examination revealed an occupying lesion in the right atrium that was producing a partial dynamic tricuspid obstruction. In addition, the chief complaint was the intermittent chest pain accompanied by a sense of impending doom following activity. Unfortunately, this symptom was misinterpreted due to the RAM causing tricuspid valve obstruction. On the basis of these findings, the case was misdiagnosed as RAM with obstruction in right ventricular effluent tract.

The surgery was conducted as quickly as possible, in anticipation of removing the myxoma and avoiding the possibility of a dangerous pulmonary embolization. Unexpectedly, the occupying mass was found not to be RAM, but a hemorrhagic cyst.

In retrospect, it is interesting to speculate about what led to the incorrect diagnosis. Firstly, the right CAF drained to the myocardial void in the current case, and not to the right atria nor the right ventricle. A hemorrhagic cyst was formed, which caused the obstruction of the CAF; subsequently, a characteristic murmur was not present. Secondly, as the large cyst slanted towards the right atrium and had no blood flow, it was easily misdiagnosed as RAM based on the echocardiogram. Thirdly, the reason for the chest pain was coronary steal syndrome in the early stage of illness, and the myocardial injury caused by the hemorrhagic cyst in the advanced stages of the disease. Unfortunately, an error was made during interpretation of this. Finally, not enough attention was paid to the ST-segment depression detected by the right precordial leads on ECG. All the aforementioned evidence suggests that patients may present with certain symptoms of right ventricular myocardial ischemia, which may lead to misdiagnosis.
Although echocardiography, due to its quickness, effectiveness and non-invasive nature, has been used as major screening tool to determine the presence, absence or status of heart disease, misdiagnosis occurs frequently due to the suboptimal acoustic window and the operator-dependent evaluation, particularly with anomalies in the coronary artery (10). The selective coronary arteriography examination remains the gold standard for the diagnosis, particularly for patients who have atypical angina, and is essential to plan surgical or percutaneous intervention (3). However, angiography may not always be possible for all individuals, as it is invasive and expensive (18). Furthermore, in this case, the echocardiogram indicated an occupying mass in the right atrium initially, and angiography may be dangerous due to the risk of rupturing the mass and causing pulmonary embolization (19). If further examinations had been made by magnetic resonance imaging or multi-slice computed tomography angiography, it may have been possible to make a differential and accurate diagnosis $(1,10)$.

In summary, the current study describes the case of a right CAF misdiagnosed as RAM. To the best of our knowledge, this is the first report of a right CAF draining to the myocardial void. To avoid misdiagnosis, thorough examination of patients is required, particularly coronary angiography. The present case additionally demonstrates how to perform CAF cardiac surgical treatment. Although there is little evidence proving that this surgical approach is more reasonable than percutaneous or surgical closure, the present study provides a potential alternative surgical method for the treatment of CAF.

\section{References}

1. Yoshitake I, Hata M, Sezai A, Niino T, Unosawa S, Shimura K, Kasamaki Y and Minami K: Cardiac angiosarcoma with cardiac tamponade diagnosed as a ruptured aneurysm of the sinus valsalva. Jpn J Clin Oncol 39: 612-615, 2009.

2. Lebreiro A, Pinho T, Silva JC, Madureira A, Macedo F, Ramos I and Maciel MJ: Percutaneous closure of a giant coronary artery fistula draining into superior vena cava. Rev Port Cardiol 29: 433-437, 2010 (In Portuguese).

3. Ogden JA: Congenital anomalies of the coronary arteries. Am J Cardiol 25: 474-479, 1970.

4. Dehmer GJ, Blankenship JC, Cilingiroglu M, Dwyer JG, Feldman DN, Gardner TJ, Grines CL, Singh M; Society for Cardiovascular Angiography and Interventions; American College of Cardiology; American Heart Association: SCAI/ACC/AHA expert consensus document: 2014 update on percutaneous coronary intervention without on-site surgical backup. Catheter Cardiovasc Interv 84: 169-187, 2014.

5. Azevedo O, Almeida J, Nolasco T, Medeiros R, Casanova J, Bartosch C, Almeida J and Pinho P: Massive right atrial myxoma presenting as syncope and exertional dyspnea: Case report. Cardiovase Ultrasound 8: 23, 2010.

6. Cho HJ, Seol SH, Choi BJ, Park SH, Kim DK, Kim U, Yang TH, Kim DK, Kim DI and Kim DS: A case of a right atrial and inferior vena caval thrombus resembling a right atrial myxoma. J Cardiovasc Ultrasound 18: 58-61,2010.

7. Demir M, Akpinar O and Acarturk E: Atrial myxoma: An unusual cause of myocardial infarction. Tex Heart Inst J 32: 445-447, 2005.

8. Berdajs DA, Mosbahi S, Charbonnier D, Hullin R and von Segesser LK: Analysis of flow dynamics in right ventricular outflow tract. J Surg Res 197: 50-57, 2015.

9. Gürbüz A, Yetkin U, Tetik O, Kestelli M and Yesil M: Right coronary artery fistula draining into the right atrium and associated with mitral valve stenosis: A case report. Heart Surg Forum 10: E325-E328, 2007.

10. Gulati GS, Ramamurthy S and Sharma S: Utility of multislice computed tomography in the diagnosis of a right coronary artery fistula to the right atrium. J Postgrad Med 53: 191-192, 2007. 
11. Benlafqih C, Léobon B, Chabbert V and Glock Y: Surgical exclusion of a symptomatic circumflex coronary to right atrium fistula. Interact Cardiovasc Thorac Surg 6: 413-414, 2007.

12. Gradaus F, Peters AJ, Schoebel FC, Gradaus D, Leschke M and Strauer BE: Angina pectoris in 'coronary steal syndrome' caused by a coronary fistula in the left ventricle. Dtsch Med Wochenschr 123: 1030-1034, 1998 (In German).

13. Rein AJ, Yatsiv I and Simcha A: An unusual presentation of right coronary artery fistula. Br Heart J 59: 598-600, 1988.

14. Said SA: Current characteristics of congenital coronary artery fistulas in adults: A decade of global experience. World J Cardiol 3: 267-277, 2011.

15. Nakamura M, Matsuoka H, Kawakami H, Komatsu J, Itou T, Higashino H, Kido T and Mochizuki T: Giant congenital coronary artery fistula to left brachial vein clearly detected by multidetector computed tomography. Circ J 70: 796-799, 2006.
16. Okamura T, Nagashima M, Yamada Y, Hiramatsu $T$ and Yamazaki K: Effective long-term surgical management of congenital coronary artery fistulas. Tohoku J Exp Med 223: 205-209, 2011.

17. Ceresnak S, Gray RG, Altmann K, Chen JM, Glickstein JS and Hellenbrand WE: Coronary artery fistulas: A review of the literature and presentation of two cases of coronary fistulas with drainage into the left atrium. Congenit Heart Dis 2: 208-213, 2007.

18. Iglesias JF, Roguelov C, Kabir T, Vogt P and Eeckhout E: Indications for urgent coronary angiography. Part I: ST-segment elevation acute coronary syndromes. Rev Med Suisse 5: 1195-1196, 1198-1201, 2009 (In French).

19. Kazuno K, Akasaka N, Kiyokawa K and Sasajima T: Ruptured aneurysm of coronary artery-to-pulmonary artery fistula. Asian Cardiovasc Thorac Ann 20: 324-326, 2012. 